\title{
Regular vector lattices of continuous functions and Korovkin-type theorems-Part I
}

\author{
by \\ Francesco Altomare and Mirella Cappelletti Montano (Bari)
}

\begin{abstract}
We introduce and study a new class of locally convex vector lattices of continuous functions on a locally compact Hausdorff space, which we call regular vector lattices. We investigate some general properties of these spaces and of the subspaces of so-called generalized affine functions. Moreover, we present some Korovkin-type theorems for continuous positive linear operators; in particular, we study Korovkin subspaces for finitely defined operators, for the identity operator and for positive projections.

Due to its length, the paper is split up into two parts of distinct character; in this first part, we introduce the class of regular vector lattices, we prove an integral representation theorem for continuous positive linear forms and we study some enveloping functions related to a continuous positive operator, together with the corresponding space of generalized affine functions. Finally, we obtain a Stone-Weierstrass type theorem.
\end{abstract}

In the second part, which will appear in the same journal, we will present some Korovkin-type theorems, together with some applications.

1. Introduction. Since their discovery, the classical theorems of Korovkin on approximation of continuous functions on a compact interval (see [22]) have impressed many mathematicians by their simplicity. Several authors have undertaken the program of extending these theorems to other settings and, in the last forty years, many interesting results have been found; this research field is often referred to as Korovkin-type approximation theory.

A quite complete picture of what has been achieved in this field up to 1996 can be found in the monographs by F. Altomare and M. Campiti ([3]; see, in particular, Appendix D), K. Donner ([14]) and G. G. Lorentz, M. von Golitschek and Y. Makovoz ([23, Chapter 16]).

More recent results that could be compared with those of this paper can be found in [4]-[6], [27].

2000 Mathematics Subject Classification: 46E05, 46E10, 46E99.

Key words and phrases: vector lattice of continuous functions, integral representation theorem, generalized affine function, Choquet boundary, Stone-Weierstrass theorem. 
With the aim to extend and to treat in a more systematic way some old and more recent results, in this paper we develop the main aspects of the Korovkin-type approximation theory in the framework of a class of locally convex vector lattices of continuous functions on a locally compact Hausdorff space $X$, which we call regular vector lattices on $X$.

Although several results obtained in the setting of abstract locally convex vector lattices are available in the literature (see, e.g., [14]-[16], [18]), our approach here is more direct and simple and gives, in addition, new results.

Examples of regular vector lattices include weighted function spaces (in particular, the space $C_{0}(X, \mathbb{R})$ of all continuous real-valued functions on $X$ vanishing at infinity) and every sublattice of $C(X, \mathbb{R})$ containing the continuous functions with compact support endowed with the topology of pointwise convergence on $X$ or with the topology of uniform convergence on compact subsets of $X$. Therefore, our results generalize and/or add some new aspects to those of F. Altomare and M. Campiti (see [3]) and H. Bauer and K. Donner (see [10]) for $C_{0}(X, \mathbb{R})$, W. Roth (see [27]) for weighted function spaces, H. Bauer (see [8], [9]), M W. Grossman (see [20]) and F. Altomare and M. Cappelletti Montano (see [4]) for adapted spaces of continuous functions (see [13], [24], [31]).

Due to its length, the paper is split up into two parts of distinct character. In this first part, we present some preliminary results on regular vector lattices. In particular, we obtain an integral representation theorem for continuous positive linear forms and, by means of some suitable enveloping functions related to a continuous positive linear operator, we study the corresponding space of generalized affine functions.

Moreover, we present a natural notion of Choquet boundary and we characterize it by means of those enveloping functions.

The case of the identity operator is also considered and, as a consequence, a Stone-Weierstrass type theorem is obtained.

In the second part of the paper, by using the results of the first part, given a regular vector lattice $(E, \tau)$ and a continuous positive linear operator $T: E \rightarrow E$, we characterize those subspaces $H$ of $E$ which are Korovkin subspaces in $E$ for $T$ and $\tau$, in the sense that every equicontinuous net of positive linear operators from $E$ into $E$ converging to $T$ on $H$ in $(E, \tau)$, automatically converges to $T$ in $(E, \tau)$.

By using this characterization, we give simple methods to construct Korovkin subspaces for particular positive linear operators, called finitely defined operators, as well as for the identity operator. We also exhibit examples of finite-dimensional Korovkin subspaces for these operators. 
Finally, we present some Korovkin-type theorems for continuous positive projections. This last part of the paper is a generalization of a previous work of the authors (see [4]) concerning the setting of adapted spaces.

\section{Regular vector lattices of continuous functions and repre-} sentation theorems. Let $X$ be a locally compact Hausdorff space. We denote by $\mathbb{R}^{X}$ and $C(X, \mathbb{R})$ the spaces of all real-valued functions on $X$ and of all continuous real-valued functions on $X$, respectively. We also denote by $C_{\mathrm{c}}(X, \mathbb{R})$ the subspace of $C(X, \mathbb{R})$ consisting of all continuous functions on $X$ whose support is compact, and by $C_{\mathrm{b}}(X, \mathbb{R})$ the subspace of all continuous real-valued bounded functions on $X$. Finally, $C_{0}(X, \mathbb{R})$ denotes the space of all continuous real-valued functions on $X$ which vanish at infinity.

Let $B_{X}$ be the $\sigma$-algebra of all Borel subsets of $X$ and denote by $M^{+}(X)$ and $M_{\mathrm{b}}^{+}(X)$ the cones of all regular (resp., regular and bounded) Borel measures on $X$. For every $x \in X$ we denote by $\varepsilon_{x}$ the Dirac measure concentrated at $x$.

If $\mu \in M^{+}(X)$, then $\operatorname{supp}(\mu)$ denotes the support of $\mu$, i.e. the complement of the largest open subset of $X$ on which $\mu$ is zero.

For every $\mu \in M^{+}(X)$ and $p \in\left[1,+\infty\right.$ [, we denote by $\mathcal{L}^{p}(X, \mu)$ the space of all Borel-measurable functions $f \in \mathbb{R}^{X}$ which are $p$-fold $\mu$-integrable.

In the particular case where $X$ is a Lebesgue-measurable subset of $\mathbb{R}^{n}$ and $\mu$ is the Lebesgue measure on $X$, we simply use the symbol $\mathcal{L}^{p}(X)$.

Let $(E, \tau)$ be a topological vector space. We denote by $(E, \tau)^{\prime}$ the space of all $\tau$-continuous linear forms on $E$. If in addition $(E, \tau)$ is a topological vector lattice, then we denote by $(E, \tau)_{+}^{\prime}$ the cone of all $\tau$-continuous positive linear forms on $E$.

We now recall a very useful result, due to B. Anger and J. Lembcke (see [7]), that generalizes the Hahn-Banach theorem to the setting of mappings that may attain the value $+\infty$.

To this end, we also recall that a mapping $p: E \rightarrow \mathbb{R} \cup\{+\infty\}$ defined on a real vector space $E$ is said to be hypolinear if

(i) $p(f+g) \leq p(f)+p(g)$ for every $f, g \in E$;

(ii) $p(\lambda f)=\lambda p(f)$ for every $f \in E$ and $\lambda \geq 0$, with the convention $0 \cdot(+\infty)=0$.

TheOREM 2.1. Let $(E, \tau)$ be a real locally convex Hausdorff space and $p: E \rightarrow \mathbb{R} \cup\{+\infty\}$ a hypolinear lower semicontinuous mapping. Then for every $f \in E$ and $\alpha \in]-p(-f), p(f)\left[\right.$ there exists $\varphi \in(E, \tau)^{\prime}$ such that $\varphi \leq p$ and $\varphi(f)=\alpha$.

If $E$ and $F$ are real vector lattices or Riesz spaces (see, e.g., [1], [28] for more details) then a (positive) linear operator $T: E \rightarrow F$ is said to be a 
lattice homomorphism if

$$
T(|f|)=|T(f)| \quad \text { for every } f \in E ;
$$

we also point out that $T$ is a lattice homomorphism if and only if

$$
T(\sup (f, g))=\sup (T(f), T(g)) \quad \text { for every } f, g \in E
$$

or, equivalently,

$$
T(\inf (f, g))=\inf (T(f), T(g)) \quad \text { for every } f, g \in E .
$$

A subset $V$ of a vector lattice $E$ is said to be a solid set (resp., a sublattice) if for every $f \in E$ and $g \in V$ such that $|f| \leq|g|$, we have $f \in V$ (resp., if $\sup (f, g) \in V$ for every $f, g \in V)$.

We now introduce a class of locally convex vector lattices of continuous functions, in which we shall develop our results.

Definition 2.2. Let $X$ be a locally compact Hausdorff space and $E$ a subspace of $C(X, \mathbb{R})$, endowed with the natural (pointwise) order $\leq$ and with a locally convex topology $\tau$.

The locally convex space $(E, \tau)$ is said to be a regular locally convex vector lattice on $X$, for short, a regular vector lattice, if

(i) $(E, \tau, \leq)$ is a locally convex vector lattice with a neighborhood base $\mathcal{U}_{\tau}$ consisting of absolutely convex solid sublattices;

(ii) $\tau$ is finer than the topology of pointwise convergence on $X$;

(iii) $C_{\mathrm{c}}(X, \mathbb{R})$ is dense in $(E, \tau)$.

We point out that property (i) is equivalent to requiring that the topology $\tau$ is generated by a saturated family $\left(p_{\alpha}\right)_{\alpha \in A}$ of seminorms on $E$ such that for every $\alpha \in A$,

(1) $p_{\alpha}(f) \leq p_{\alpha}(g)$ for every $f, g \in E,|f| \leq|g|$;

(2) $p_{\alpha}(\sup (f, g))=\sup \left(p_{\alpha}(f), p_{\alpha}(g)\right)$ for every $f, g \in E_{+}$.

Such seminorms are also called $M$-seminorms. Below we present some examples of regular vector lattices.

EXAMPLES 2.3. 1. Every sublattice $E$ of $C(X, \mathbb{R})$ containing $C_{\mathrm{c}}(X, \mathbb{R})$ and endowed with the topology $\tau_{\mathrm{c}}$ of uniform convergence on compact subsets of $X$ or the topology $\tau_{\mathrm{s}}$ of pointwise convergence on $X$ is a regular vector lattice.

We recall that a neighborhood base of the origin for $\tau_{\mathrm{c}}$ (resp., $\tau_{\mathrm{s}}$ ) is

$$
\mathcal{U}_{c}:=\left\{V_{K, \varepsilon} \mid \varepsilon>0, K \text { compact }\right\},
$$

(resp.,

$$
\left.\mathcal{U}_{s}:=\left\{V_{F, \varepsilon} \mid \varepsilon>0, F \text { finite }\right\}\right),
$$

where, for $Q \subset X$ and $\varepsilon>0$, we set

$$
V_{Q, \varepsilon}:=\{f \in E|| f(x) \mid \leq \varepsilon \text { for every } x \in Q\} .
$$


2. Let $H$ be an adapted subspace of $C(X, \mathbb{R})$ (see [13, Vol. II, Ch. 8], [24], [31]) and set

$$
E_{H}:=\{f \in C(X, \mathbb{R}) \mid \text { there exists } h \in H \text { such that }|f| \leq h\} .
$$

Then $E_{H}$ is a sublattice of $C(X, \mathbb{R})$ containing $C_{\mathrm{c}}(X, \mathbb{R})([13$, Vol. II, Remark 34.5, Theorem 34.6]). Accordingly, the subspace $E_{H}$ endowed with the topologies $\tau_{\mathrm{s}}$ and $\tau_{\mathrm{c}}$, respectively, is a regular vector lattice.

Several examples of adapted spaces are indicated in [13], [24], [31].

Here we limit ourselves to pointing out that, if $X$ is a locally compact subset of $\mathbb{R}^{n}$, then the subspace $H$ of all real-valued polynomials on $X$ is adapted; moreover

$$
\begin{gathered}
E_{H}=\left\{f \in C(X, \mathbb{R}) \mid \text { there exist } m \in \mathbb{N} \text { and } \alpha, \beta \in \mathbb{R}^{+}\right. \text {such that } \\
\left.\left|f\left(x_{1}, \ldots, x_{n}\right)\right| \leq \alpha\left|x_{1}\right|^{m} \cdots\left|x_{n}\right|^{m}+\beta \text { for every }\left(x_{1}, \ldots, x_{n}\right) \in X\right\} .
\end{gathered}
$$

3 (Weighted function spaces). Let $W$ be a family of positive upper semicontinuous functions on $X$ such that if $w_{1}, w_{2} \in W$ then there exist $w \in W$ and $\alpha>0$ such that $w_{1} \leq \alpha w$ and $w_{2} \leq \alpha w$. Further, assume that for every $x \in X$ there exists $w \in W$ such that $w(x)>0$.

Consider the weighted function space $C_{W}(X, \mathbb{R})$ of all functions $f \in$ $C(X, \mathbb{R})$ such that $w f$ vanishes at infinity for all $w \in W$.

The space $C_{W}(X, \mathbb{R})$ is endowed with the locally convex topology $\tau_{W}$ generated by the family $\left(p_{w}\right)_{w \in W}$ of seminorms defined by

$$
p_{w}(f):=\sup _{x \in X} w(x)|f(x)| \quad(f \in E)
$$

(see [25], [26]). Then $\left(C_{W}(X, \mathbb{R}), \tau_{W}\right)$ is a regular vector lattice on $X$.

Indeed, properties (i)-(iii) are obvious (see, e.g., [26]), upon taking into account that a neighborhood base of the origin is

$$
\mathcal{U}_{W}:=\left\{V_{w, \varepsilon} \mid w \in W, \varepsilon>0\right\}
$$

where

$$
V_{w, \varepsilon}:=\left\{f \in C_{W}(X, \mathbb{R}) \mid p_{w}(f) \leq \varepsilon\right\} \quad(w \in W, \varepsilon>0) .
$$

We give some examples of weighted function spaces that can be useful for more concrete applications.

3.a. The space $C_{\mathrm{c}}(X, \mathbb{R})$ is the weighted function space $C_{W}(X, \mathbb{R})$, where $W=\{w \in C(X, \mathbb{R}) \mid w \geq 0\}$. The topology $\tau_{W}$ is coarser than the inductive limit topology on $C_{\mathrm{c}}(X, \mathbb{R})$ and finer than the topology of uniform convergence on $X$.

3.b. The space $C_{0}(X, \mathbb{R})$ is the weighted function space $C_{W}(X, \mathbb{R})$, where $W=\{\mathbf{1}\}$. The topology $\tau_{W}$ is the topology of uniform convergence on $X$.

3.c. The space $C_{\mathrm{b}}(X, \mathbb{R})$ is the weighted function space $C_{W}(X, \mathbb{R})$, where $W=\left\{w \in C_{0}(X, \mathbb{R}) \mid w \geq 0\right\}$. Its topology $\tau_{W}$ is called the strict topology 
and it is finer than the topology of uniform convergence on compact subsets of $X$ and coarser than the topology of uniform convergence on $X$.

3.d. The space $C(X, \mathbb{R})$ endowed with the topology of uniform convergence on compact subsets of $X$ (resp., the topology of pointwise convergence on $X)$ is the weighted function space $C_{W_{\mathrm{c}}}(X, \mathbb{R})$ (resp., $C_{W_{\mathrm{s}}}(X, \mathbb{R})$ ), where $W_{\mathrm{c}}$ consists of the characteristic functions of all compact subsets of $X$ (resp., $W_{\mathrm{s}}$ consists of the characteristic functions of all finite subsets of $X$ ).

Coming back to the general definition, we point out that if all the functions in $W$ are strictly positive and $W$ is filtering decreasing, then $C_{W}(X, \mathbb{R})$ is also an adapted space (see [31, Prop. 4 and Corollary 5, pp. 14-15]). On the other hand, the space $C_{\mathrm{b}}(X, \mathbb{R})$ is not adapted.

4. Assume that $X$ is noncompact and denote by $C_{*}(X, \mathbb{R})$ the subspace of all functions in $C(X, \mathbb{R})$ which are convergent at the point at infinity $\infty$ of $X$. Then $C_{*}(X, \mathbb{R})$, endowed with the natural order and the topology of uniform convergence on $X$, is not a regular vector lattice (property (iii) is not satisfied).

A useful property of regular vector lattices is shown below. We first prove a preliminary lemma.

LEMMA 2.4. Let $X$ be a locally compact Hausdorff space and $(E, \tau)$ a regular vector lattice on $X$. If $\varrho \in(E, \tau)_{+}^{\prime}$ is a lattice homomorphism on $E$, then there exist $x \in X$ and $\lambda \geq 0$ such that $\varrho(f)=\lambda f(x)$ for every $f \in E$.

Proof. Consider the (unique) $\mu \in M^{+}(X)$ such that

$$
\varrho(f)=\int f d \mu \quad \text { for every } f \in C_{\mathrm{c}}(X, \mathbb{R}) .
$$

Accordingly, for every $f \in C_{\mathrm{c}}(X, \mathbb{R})$ we get

$$
\left|\int f d \mu\right|=\int|f| d \mu \text {. }
$$

We shall show that there exist $\lambda \geq 0$ and $x \in X$ such that $\mu=\lambda \varepsilon_{x}$. In fact, if $\mu=0$, then choose $\lambda=0$. If $\mu \neq 0$, then $\operatorname{supp}(\mu) \neq \emptyset$. Take $x \in \operatorname{supp}(\mu)$ and $\varphi \in C_{\mathrm{c}}(X, \mathbb{R}), 0 \leq \varphi \leq 1$, such that $\varphi(x)=1$. Then $\alpha:=\int \varphi d \mu>0$; otherwise, we would have $\varphi=0$ on $\operatorname{supp}(\mu)$, so that $\varphi(x)=0$.

To prove that $\operatorname{supp}(\mu)=\{x\}$, choose $f \in C_{\mathrm{c}}(X, \mathbb{R})$ such that $\operatorname{supp}(f) \subset$ $X \backslash\{x\}$; we shall show that $\int f d \mu=0$.

Otherwise, setting $\beta:=\int f d \mu$, we would get

$$
\int \frac{1}{\beta} f d \mu=\int \frac{1}{\alpha} \varphi d \mu=1
$$

so from (2) it would follow that

$$
0=\left|\int \frac{1}{\beta} f-\frac{1}{\alpha} \varphi d \mu\right|=\int\left|\frac{1}{\beta} f-\frac{1}{\alpha} \varphi\right| d \mu \text {. }
$$


Accordingly, $(1 / \beta) f-(1 / \alpha) \varphi=0$ on $\operatorname{supp}(\mu)$, but this is impossible since $(1 / \beta) f(x)-(1 / \alpha) \varphi(x)=-1 / \alpha \neq 0$.

Hence, from (1) it follows that

$$
\varrho(f)=\int f d \mu=\lambda f(x) \quad\left(f \in C_{\mathrm{c}}(X, \mathbb{R})\right) .
$$

Fix now $f \in E$; since by (iii) of Definition $2.2, C_{\mathrm{c}}(X, \mathbb{R})$ is dense in $(E, \tau)$, there exists a net $\left(f_{i}\right)_{i \in I}^{\leq}$in $C_{\mathrm{c}}(X, \mathbb{R})$ such that $\lim _{i \in I} \leq f_{i}=f$ in $(E, \tau)$. By (ii) of Definition 2.2, we also get $\lim _{i \in I} \leq f_{i}(x)=f(x)$; hence

$$
\varrho(f)=\lim _{i \in I} \leq \varrho\left(f_{i}\right)=\lim _{i \in I} \leq \lambda f_{i}(x)=\lambda f(x)
$$

and this completes the proof.

Proposition 2.5. Every regular vector lattice $(E, \tau)$ on a locally compact Hausdorff space $X$ has Dini's property, i.e. if $\left(f_{i}\right)_{i \in I}^{\leq}$is a filtering decreasing net in $E$ such that $\inf _{i \in I} f_{i}=0$, then $\lim _{i \in I} \leq f_{i}=0$ in $(E, \tau)$.

Proof. Indeed, if $(E, \tau)$ is a regular vector lattice on $X$, it is an $M$-space in the sense of [21, Section 4.3], so that it is a Dini lattice, i.e. every filtering decreasing net in $E$ converging to 0 pointwise on $V(E, \tau)$, converges to 0 with respect to $\tau$, where $V(E, \tau)$ denotes the set of all real-valued $\tau$-continuous lattice homomorphisms on $E$ (see [17, p. 180]).

Hence, our statement follows from Lemma 2.4.

The following integral representation property will be essential for our work. It generalizes similar results obtained in the framework of Examples 2.3 (see [12], [32]).

THEOREM 2.6. Let $X$ be a locally compact Hausdorff space and $(E, \tau)$ a regular vector lattice on $X$. Then for every $\varrho \in(E, \tau)_{+}^{\prime}$, there exists a (unique) $\mu \in M^{+}(X)$ such that $E \subset \mathcal{L}^{1}(X, \mu)$ and

$$
\varrho(f)=\int f d \mu \quad \text { for every } f \in E .
$$

Proof. From the Riesz representation theorem, there exists $\mu \in M^{+}(X)$ such that

$$
\varrho(\varphi)=\int \varphi d \mu \quad \text { for every } \varphi \in C_{\mathrm{c}}(X, \mathbb{R}) .
$$

Fix now $f \in E, f \geq 0$; then for every $\varphi \in C_{\mathrm{c}}(X, \mathbb{R})$ with $0 \leq \varphi \leq f$,

$$
\int \varphi d \mu=\varrho(\varphi) \leq \varrho(f) .
$$

Moreover, set

$$
\mathcal{A}_{f}:=\left\{\varphi \in C_{\mathrm{c}}(X, \mathbb{R}) \mid 0 \leq \varphi \leq f\right\} .
$$

An easy application of Urysohn's lemma shows that

$$
f=\sup _{\varphi \in \mathcal{A}_{f}} \varphi .
$$


Since $\mathcal{A}_{f}$ is filtering increasing, from Proposition 2.5 we get

$$
f=\sup _{\varphi \in \mathcal{A}_{f}} \varphi=\lim _{\varphi \in \mathcal{A}_{f}} \leq \varphi \quad \text { in }(E, \tau) .
$$

On the other hand, from [11, Theorem 1.5], it follows that

$$
\int f d \mu=\sup _{\varphi \in \mathcal{A}_{f}} \int \varphi d \mu \leq \varrho(f)<\infty ;
$$

accordingly, $f \in \mathcal{L}^{1}(X, \mu)$ and

$$
\int f d \mu=\sup _{\varphi \in \mathcal{A}_{f}} \int \varphi d \mu=\sup _{\varphi \in \mathcal{A}_{f}} \varrho(\varphi)=\lim _{\varphi \in \mathcal{A}_{f}} \leq \varrho(\varphi)=\varrho(f) .
$$

Since $E=E_{+}-E_{+}$, this completes the proof.

REMARK 2.7. We point out that, if $(E, \tau, \leq)$ is a locally convex vector sublattice of $C(X, \mathbb{R})$ satisfying property (i) of Definition 2.2 , and an integral representation theorem such as Theorem 2.6 holds in $E$, then necessarily $C_{\mathrm{c}}(X, \mathbb{R})$ is dense in $(E, \tau)$.

Otherwise, there would exist $\varrho \in(E, \tau)^{\prime}$ such that $\varrho \neq 0$ and $\varrho=0$ on $C_{\mathrm{c}}(X, \mathbb{R})$. By $\left[1\right.$, Theorem 5.7], there would exist $\varrho_{1}, \varrho_{2} \in(E, \tau)_{+}^{\prime}$ such that $\varrho=\varrho_{1}-\varrho_{2}$ and, consequently, there would exist $\mu_{1}, \mu_{2} \in M^{+}(X)$ such that for every $f \in E$,

$$
\varrho_{1}(f)=\int f d \mu_{1}, \quad \varrho_{2}(f)=\int f d \mu_{2} .
$$

In particular, for every $\varphi \in C_{\mathrm{c}}(X, \mathbb{R})$,

$$
\int \varphi d \mu_{1}=\int \varphi d \mu_{2}
$$

so that $\mu_{1}=\mu_{2}$. Accordingly, $\varrho_{1}=\varrho_{2}$ and hence $\varrho=0$, a contradiction.

Finally, we state a property which holds true in a more general context of vector lattices.

Proposition 2.8. Let $X$ be a locally compact Hausdorff space, $(E, \tau, \leq)$ a locally convex vector sublattice of $C(X, \mathbb{R})$ satisfying property (i) of Definition 2.2 , and assume that $C_{\mathrm{c}}(X, \mathbb{R}) \subset E$. Then for every $f \in E$ and $x \in X$ and for every $V \in \mathcal{U}_{\tau}$ there exists a neighborhood $Q$ of $x$ such that

$$
|f-f(x)| \varphi \in V
$$

for every $\varphi \in C_{\mathrm{c}}(X, \mathbb{R})$ with $0 \leq \varphi \leq 1$ and $\operatorname{supp}(\varphi) \subset Q$.

Proof. Fix $f \in E, x \in X$ and $V \in \mathcal{U}_{\tau}$ and choose a compact neighborhood $Q_{0}$ of $x$. Moreover, consider a function $\varphi_{Q_{0}} \in C_{\mathrm{c}}(X, \mathbb{R}), 0 \leq \varphi_{Q_{0}} \leq 1$, such that $\varphi_{Q_{0}}=1$ on $Q_{0}$.

There exists $\varepsilon>0$ such that $\varepsilon \varphi_{Q_{0}} \in V$. Further, since $f$ is continuous, there exists a neighborhood $Q \subset Q_{0}$ of $x$ such that for every $y \in Q$,

$$
|f(y)-f(x)| \leq \varepsilon .
$$


For every $\varphi \in C_{\mathrm{c}}(X, \mathbb{R})$ with $0 \leq \varphi \leq 1$ and $\operatorname{supp}(\varphi) \subset Q$, we clearly have $\varphi \leq \varphi_{Q_{0}}$. Hence, for every $y \in Q$,

$$
|f(y)-f(x)| \varphi(y) \leq \varepsilon \varphi_{Q_{0}}(y),
$$

while for every $y \notin Q$,

$$
|f(y)-f(x)| \varphi(y)=0 \leq \varepsilon \varphi_{Q_{0}}(y) .
$$

Accordingly,

$$
0 \leq|f-f(x)| \varphi \leq \varepsilon \varphi_{Q_{0}} \in V
$$

so that, $V$ being solid, it follows that $|f-f(x)| \varphi \in V$.

3. Generalized affine functions and Choquet boundaries. In this section, we introduce some enveloping functions for each function of a regular vector lattice and we study the corresponding space of generalized affine functions. Moreover, we introduce a natural notion of Choquet boundary and we characterize it by means of those enveloping functions.

From now on, we fix a locally compact Hausdorff space $X$ and a regular vector lattice $(E, \tau)$ on $X$.

For every $\mu \in M^{+}(X)$ such that $E \subset \mathcal{L}^{1}(X, \mu)$, consider the positive linear form $I_{\mu}: E \rightarrow \mathbb{R}$ defined by

$$
I_{\mu}(f):=\int f d \mu \quad(f \in E)
$$

and set

$$
M_{\tau, E}^{+}(X):=\left\{\mu \in M^{+}(X) \mid E \subset \mathcal{L}^{1}(X, \mu) \text { and } I_{\mu} \in(E, \tau)_{+}^{\prime}\right\} .
$$

By Theorem 2.6, $M_{\tau, E}^{+}(X)$ can be identified with $(E, \tau)_{+}^{\prime}$. In fact some definitions and results of this paper could be stated and proved by replacing $M_{\tau, E}^{+}(X)$ with $(E, \tau)_{+}^{\prime}$. On the other hand, in other situations, e.g. when studying Choquet boundaries, the use of Borel measures is essential. Hence, to get more complete results, we prefer to use Borel measures everywhere.

We finally point out that, if $\left(p_{\alpha}\right)_{\alpha \in A}$ is a saturated family of $M$-seminorms, then a Borel measure $\mu \in M^{+}(X)$ belongs to $M_{\tau, E}^{+}(X)$ if and only if there exist $c \geq 0$ and $\alpha \in A$ such that for every $\varphi \in C_{\mathrm{c}}(X, \mathbb{R})$,

$$
\left|\int \varphi d \mu\right| \leq c p_{\alpha}(\varphi) \text {. }
$$

Indeed, if $\mu$ satisfies the inequality above, then the functional $I_{\mu}^{*}: C_{\mathrm{c}}(X, \mathbb{R})$ $\rightarrow \mathbb{R}$ defined by $I_{\mu}^{*}(\varphi)=\int \varphi d \mu\left(\varphi \in C_{\mathrm{c}}(X, \mathbb{R})\right)$ is $\tau$-continuous and hence it can be continuously extended to a continuous linear form $\varrho$ on $E$. If $f \in E$ and $f \geq 0$, then $f=\sup _{\varphi \in \mathcal{A}_{f}} \varphi=\lim _{\varphi \in \mathcal{A}_{f}} \leq \varphi$ (see the proof of Theorem 2.6) and so

$$
\int f d \mu=\sup _{\varphi \in \mathcal{A}_{f}} \int \varphi d \mu=\lim _{\varphi \in \mathcal{A}_{f}} \int \varphi d \mu=\varrho(f) .
$$


Therefore $f \in \mathcal{L}^{1}(X, \mu)$. This shows that $E=E_{+}-E_{+} \subset \mathcal{L}^{1}(X, \mu)$ and $I_{\mu}=\varrho$ is $\tau$-continuous, i.e. $\mu \in M_{\tau, E}^{+}(X)$. The converse implication is obvious.

Fix now a subspace $H$ of $E$ and for every $\mu \in M_{\tau, E}^{+}(X)$ consider the mappings $\widehat{\mu}_{\tau, H}: E \rightarrow \mathbb{R} \cup\{+\infty\}$ and $\widetilde{\mu}_{\tau, H}: E \rightarrow \mathbb{R} \cup\{-\infty\}$ defined by

$$
\begin{aligned}
& \widehat{\mu}_{\tau, H}(f):=\sup _{U \in \mathcal{U}_{\tau}} \inf _{\substack{k \in H \\
(f-k)^{+} \in U}} \int k d \mu, \\
& \widetilde{\mu}_{\tau, H}(f):=\inf _{U \in \mathcal{U}_{\tau}} \sup _{\substack{h \in H \\
(h-f)^{+} \in U}} \int h d \mu,
\end{aligned}
$$

for every $f \in E$, with the conventions $\inf \emptyset=+\infty$ and $\sup \emptyset=-\infty$.

We point out that if we set

$H_{\tau}^{u}:=\left\{f \in E \mid\right.$ for every $U \in \mathcal{U}_{\tau}$ there exists $k \in H$

$$
\text { such that } \left.(f-k)^{+} \in U\right\}
$$

$H_{\tau}^{l}:=\left\{f \in E \mid\right.$ for every $U \in \mathcal{U}_{\tau}$ there exists $h \in H$

$$
\text { such that } \left.(h-f)^{+} \in U\right\} \text {, }
$$

then $\widehat{\mu}_{\tau, H}(f)=+\infty$ (resp., $\left.\widetilde{\mu}_{\tau, H}(f)=-\infty\right)$ for every $f \in E \backslash H_{\tau}^{u}$ (resp., for every $\left.f \in E \backslash H_{\tau}^{l}\right)$.

We list some elementary properties of $\widehat{\mu}_{\tau, H}$ and $\breve{\mu}_{\tau, H}$, whose proofs are straightforward:

$$
\begin{aligned}
\widetilde{\mu}_{\tau, H}(h) & =\int h d \mu=\widehat{\mu}_{\tau, H}(h) & & \text { for every } h \in H \\
\widehat{\mu}_{\tau, H}(f+g) & \leq \widehat{\mu}_{\tau, H}(f)+\widehat{\mu}_{\tau, H}(g) & & \text { for every } f, g \in E \\
\widehat{\mu}_{\tau, H}(\lambda f) & =\lambda \widehat{\mu}_{\tau, H}(f) & & \text { for every } f \in E \text { and } \lambda \geq 0,
\end{aligned}
$$

with the convention $0 \cdot(+\infty)=0$;

$$
\widetilde{\mu}_{\tau, H}(f)=-\widehat{\mu}_{\tau, H}(-f) \quad \text { for every } f \in E .
$$

Proposition 3.1. For every $\mu \in M_{\tau, E}^{+}(X)$ and $f \in E$,

$$
\widetilde{\mu}_{\tau, H}(f) \leq \int f d \mu \leq \widehat{\mu}_{\tau, H}(f) \text {. }
$$

Proof. We show the second inequality; the proof of the first is similar.

If $f \in E \backslash H_{\tau}^{u}$, there is nothing to prove. Assume that $f \in H_{\tau}^{u}$; since $I_{\mu}$ is $\tau$-continuous, given $\varepsilon>0$, there exists $V \in \mathcal{U}_{\tau}$ such that $\left|\int g d \mu\right| \leq \varepsilon$ for every $g \in V$. Accordingly, for every $k \in H$ such that $(f-k)^{+} \in V$, we get

$$
\int f d \mu-\int k d \mu \leq \int(f-k)^{+} d \mu \leq \varepsilon .
$$

Therefore,

$$
\int f d \mu \leq \inf _{\substack{k \in H \\(f-k)^{+} \in V}} \int k d \mu+\varepsilon \leq \widehat{\mu}_{\tau, H}(f)+\varepsilon
$$

and, since $\varepsilon$ was arbitrarily chosen, this completes the proof. 
We are now in a position to show a fundamental property of $\widehat{\mu}_{\tau, H}$.

Proposition 3.2. The mapping $\widehat{\mu}_{\tau, H}$ is lower semicontinuous on $E$ with respect to $\tau$.

Proof. First, we observe that $H_{\tau}^{u}$ is a closed subset of $E$.

Indeed, fix $f \in \overline{H_{\tau}^{u}}$ and $U \in \mathcal{U}_{\tau}$ and consider $V \in \mathcal{U}_{\tau}$ such that $V+V \subset U$. Then there exists $g \in H_{\tau}^{u}$ with $f-g \in V$ and hence $|f-g| \in V$; moreover, there exists $h \in H$ such that $(g-h)^{+} \in V$. Since

$$
f-h=f-g+g-h \leq|f-g|+(g-h)^{+},
$$

clearly we get

$$
(f-h)^{+} \leq|f-g|+(g-h)^{+} \in V+V \subset U,
$$

so that $f \in H_{\tau}^{u}$.

To prove that $\widehat{\mu}_{\tau, H}$ is lower semicontinuous on $E$, fix $f \in E$ and consider $\alpha \in \mathbb{R}$ such that $\alpha<\widehat{\mu}_{\tau, H}(f)$.

If $f \in E \backslash H_{\tau}^{u}$, then $\alpha<\widehat{\mu}_{\tau, H}(g)=+\infty$ for every $g \in E \backslash H_{\tau}^{u}$, with $E \backslash H_{\tau}^{u}$ open. If $f \in H_{\tau}^{u}$, fix $U \in \mathcal{U}_{\tau}$ such that

$$
\alpha<\inf _{\substack{k \in H \\(f-k)^{+} \in U}} \int k d \mu .
$$

Moreover, consider $V \in \mathcal{U}_{\tau}$ such that $V+V \subset U$ and $g \in E$ with $f-g \in V$, so that $|f-g| \in V$.

If $g \notin H_{\tau}^{u}$, then $\widehat{\mu}_{\tau, H}(g)=+\infty>\alpha$; on the other hand, if $g \in H_{\tau}^{u}$, then for every $h \in H$ such that $(g-h)^{+} \in V$, we get

$$
f-h=f-g+g-h \leq|f-g|+(g-h)^{+}
$$

and hence

$$
(f-h)^{+} \leq|f-g|+(g-h)^{+} \in V+V \subset U .
$$

Therefore,

$$
\inf _{\substack{k \in H \\(f-k)^{+} \in U}} \int k d \mu \leq \int h d \mu
$$

so that

$$
\inf _{\substack{k \in H \\(f-k)^{+} \in U}} \int k d \mu \leq \inf _{\substack{h \in H \\(g-h)^{+} \in V}} \int h d \mu \leq \widehat{\mu}_{\tau, H}(g) .
$$

Thus, $\alpha<\widehat{\mu}_{\tau, H}(g)$, and this completes the proof.

Set now

$$
\begin{aligned}
& \mathcal{L}_{\tau, \tau_{\mathrm{s}}}^{+}(E):=\left\{T: E \rightarrow E \mid T \text { is a }\left(\tau, \tau_{\mathrm{s}}\right)\right. \text {-continuous } \\
& \text { positive linear operator }\} \\
& \mathcal{L}_{\tau}^{+}(E):=\{T: E \rightarrow E \mid T \text { is a }(\tau, \tau) \text {-continuous } \\
& \text { positive linear operator }\}
\end{aligned}
$$


Then for a given $T \in \mathcal{L}_{\tau, \tau_{\mathrm{s}}}^{+}(E)$ and $x \in X$ consider the positive linear form $\varrho_{x}: E \rightarrow \mathbb{R}$ defined by

$$
\varrho_{x}(f):=T(f)(x) \quad(f \in E) .
$$

Obviously, $\varrho_{x} \in(E, \tau)_{+}^{\prime}$ and hence by Theorem 2.6 there exists (a unique) $\mu_{x} \in M_{\tau, E}^{+}(X)$ such that $E \subset \mathcal{L}^{1}\left(X, \mu_{x}\right)$ and

$$
T(f)(x)=\int f d \mu_{x}
$$

for every $f \in E$. Taking (3.1) and (3.2) into account, for every $x \in X$ and $f \in E$, set

$$
\begin{aligned}
& \widehat{f}_{\tau, T}(x):=\left(\widehat{\mu}_{x}\right)_{\tau, H}(f)=\sup _{U \in \mathcal{U}_{\tau}} \inf _{\substack{k \in H \\
(f-k)^{+} \in U}} T(k)(x), \\
& \check{f}_{\tau, T}(x):=\left(\widetilde{\mu}_{x}\right)_{\tau, H}(f)=\inf _{U \in \mathcal{U}_{\tau}} \sup _{\substack{h \in H \\
(h-f)^{+} \in U}} T(h)(x) .
\end{aligned}
$$

Clearly, from Proposition 3.1, it follows that

$$
\check{f}_{\tau, T} \leq T(f) \leq \widehat{f}_{\tau, T} \quad \text { for every } f \in E,
$$

and from (3.3) we get

$$
\check{h}_{\tau, T}=T(h)=\widehat{h}_{\tau, T} \quad \text { for every } h \in H .
$$

An important role will be played by the following subspace of $E$ :

$$
\widehat{H}_{\tau, T}:=\left\{f \in E \mid \check{f}_{\tau, T}=T(f)=\widehat{f}_{\tau, T}\right\},
$$

which we shall refer to as the subspace of generalized H-affine functions with respect to the operator $T$ and the topology $\tau$.

Moreover, for every $x \in X$, consider the set

$$
M_{\tau, x}^{T}(H):=\left\{\mu \in M_{\tau, E}^{+}(X) \mid \int h d \mu=T(h)(x) \text { for every } h \in H\right\} .
$$

Every $\mu \in M_{\tau, x}^{T}(H)$ is said to be an H-representing measure for $x$ with respect to the operator $T$ and the topology $\tau$.

We point out that, given $x \in X$, from (3.11) and (3.12) it follows that for every $\mu \in M_{\tau, x}^{T}(H)$ and $f \in E$,

$$
\widehat{f}_{\tau, T}(x)=\widehat{\mu}_{\tau, H}(f), \quad \check{f}_{\tau, T}(x)=\check{\mu}_{\tau, H}(f) .
$$

Proposition 3.1 also shows that if $x \in X, \mu \in M_{\tau, x}^{T}(H)$ and $f \in E$, then

$$
\check{f}_{\tau, T}(x) \leq \int f d \mu \leq \widehat{f}_{\tau, T}(x) .
$$


Finally, the set

$$
\begin{aligned}
& \partial_{\tau, H}^{T} X:=\left\{x \in X \mid M_{\tau, x}^{T}(H)=\left\{\mu_{x}\right\}\right\} \\
&=\left\{x \in X \mid \text { if } \mu \in M_{\tau, E}^{+}(X) \text { and } \int h d \mu=T(h)(x) \text { for every } h \in H,\right. \\
&\text { then } \left.\int f d \mu=T(f)(x) \text { for every } f \in E\right\}
\end{aligned}
$$

is defined to be the Choquet boundary of $H$ with respect to the operator $T$ and the topology $\tau$.

REMARK 3.3. By (3.13) and (3.16) it is easy to check that $\partial_{\tau, H}^{T} X=$ $\partial_{\tau, \widehat{H}_{\tau, T}}^{T} X$

In what follows, we shall characterize the Choquet boundary of $H$ with respect to an operator $T \in \mathcal{L}_{\tau, \tau_{\mathrm{s}}}^{+}(E)$ in terms of the enveloping mappings (3.11) and (3.12). To this end, we state the following result.

TheOREM 3.4. Let $X$ be a locally compact Hausdorff space, $(E, \tau)$ a regular vector lattice on $X, H$ a subspace of $E$, and $T \in \mathcal{L}_{\tau, \tau_{\mathrm{s}}}^{+}(E)$. Then for every $x \in X$ and $f \in E$,

$$
] \check{f}_{\tau, T}(x), \widehat{f}_{\tau, T}(x)\left[\subset\left\{\int f d \mu \mid \mu \in M_{\tau, x}^{T}(H)\right\} \subset\left[\check{f}_{\tau, T}(x), \widehat{f}_{\tau, T}(x)\right] .\right.
$$

Proof. The second inclusion is a consequence of (3.16).

In order to show the first inclusion, note that if the open interval is empty, there is nothing to prove; so assume that it is nonempty, fix $x \in X$ and $\gamma \in] \breve{f}_{\tau, T}(x), \widehat{f}_{\tau, T}(x)[$, and consider the functional $p: E \rightarrow \mathbb{R} \cup\{+\infty\}$ defined by $p(g):=\widehat{g}_{\tau, T}(x)$ for every $g \in E$.

By (3.4), (3.5) and Proposition 3.2, $p$ is a hypolinear, $\tau$-lower semicontinuous functional. Moreover, since (3.6) holds, Theorem 2.1 yields $\varphi \in(E, \tau)^{\prime}$ such that $\varphi \leq p$ and $\varphi(f)=\gamma$. The linear form $\varphi$ is also positive because if $g \in E$ and $g \leq 0$, then $\varphi(g) \leq p(g) \leq 0$.

Therefore, from Theorem 2.6, there exists $\mu \in M^{+}(X)$ such that $E \subset$ $\mathcal{L}^{1}(X, \mu)$ and $\varphi(g)=\int g d \mu$ for every $g \in E$. Note that, for $h \in H$, we have

$$
\int h d \mu=\varphi(h) \leq p(h)=T(h)(x)
$$

and so, replacing $h$ by $-h$, we get

$$
\int h d \mu=T(h)(x) \text {. }
$$

Hence, $\mu \in M_{\tau, x}^{T}(H)$ and $\gamma=\varphi(f)=\int f d \mu$, and this completes the proof.

From Theorem 3.4 several corollaries follow.

COROLlary 3.5. Under the assumptions of Theorem 3.4,

$$
\partial_{\tau, H}^{T} X=\left\{x \in X \mid \check{f}_{\tau, T}(x)=T(f)(x)=\widehat{f}_{\tau, T}(x) \text { for every } f \in E\right\} \text {. }
$$


In particular, if $\partial_{\tau, H}^{T} X \neq \emptyset$, then $\widehat{f}_{\tau, T}(x)<+\infty$ and $\check{f}_{\tau, T}(x)>-\infty$ for every $x \in \partial_{\tau, H}^{T} X$ and $f \in E$.

In the next result, we characterize the generalized $H$-affine functions with respect to $T$ and $\tau$ by means of $H$-representing measures.

Corollary 3.6. Under the assumptions of Theorem 3.4, fix $f \in E$. Then $f \in \widehat{H}_{\tau, T}$ if and only if $\int f d \mu=T(f)(x)$ for every $x \in X$ and $\mu \in$ $M_{\tau, x}^{T}(H)$. In particular, $\partial_{\tau, H}^{T} X=X$ if and only if $\widehat{H}_{\tau, T}=E$.

Another useful characterization of the generalized $H$-affine functions is shown below.

TheOREM 3.7. Let $X$ be a locally compact Hausdorff space, $(E, \tau)$ a regular vector lattice on $X, H$ a subspace of $E, T \in \mathcal{L}_{\tau, \tau_{\mathrm{s}}}^{+}(E)$, and $f \in E$. Then the following statements are equivalent:

(a) $f \in \widehat{H}_{\tau, T}$.

(b) For every $V \in \mathcal{U}_{\tau}$ there exist $n, m \in \mathbb{N}$ and $k_{1}, \ldots, k_{n}, k_{1}^{\prime}, \ldots, k_{m}^{\prime} \in H$ such that

(i) $\left(f-k_{i}\right)^{+} \in V$ for every $i=1, \ldots, n$ and $\left(k_{j}^{\prime}-f\right)^{+} \in V$ for every $j=1, \ldots, m$

(ii) $\inf _{1 \leq i \leq n} T\left(k_{i}\right)-\sup _{1 \leq j \leq m} T\left(k_{j}^{\prime}\right) \in V$.

Proof. (a) $\Rightarrow$ (b). Fix $f \in \widehat{H}_{\tau, T}$ and consider the subset $G$ of $E$ consisting of all functions of the form

$$
T(f)-\inf _{\substack{k \in H \\(f-k)^{+} \in U}} T(k),
$$

with $U \in \mathcal{U}_{\tau}$. We show that $G$ is filtering decreasing.

Indeed, fix $U, V \in \mathcal{U}_{\tau}$ and set $W:=U \cap V$; then

$$
\inf _{\substack{k \in H \\(f-k)^{+} \in U}} T(k) \leq \inf _{\substack{k \in H \\(f-k)^{+} \in W}} T(k), \quad \inf _{\substack{k \in H \\(f-k)^{+} \in V}} T(k) \leq \inf _{\substack{k \in H \\(f-k)^{+} \in W}} T(k) .
$$

Accordingly,

$$
\begin{gathered}
T(f)-\inf _{\substack{k \in H \\
(f-k)^{+} \in W}} T(k) \leq T(f)-\inf _{\substack{k \in H \\
(f-k)^{+} \in U}} T(k), \\
T(f)-\inf _{\substack{k \in H \\
(f-k)^{+} \in W}} T(k) \leq T(f)-\inf _{\substack{k \in H \\
(f-k)^{+} \in V}} T(k),
\end{gathered}
$$

so that $G$ is filtering decreasing.

Moreover,

$$
\begin{aligned}
\inf _{g \in G} g & =\inf _{U \in \mathcal{U}_{\tau}}\left(T(f)-\inf _{\substack{k \in H \\
(f-k)^{+} \in U}} T(k)\right)=T(f)-\sup _{U \in \mathcal{U}_{\tau}} \inf _{\substack{k \in H \\
(f-k)^{+} \in U}} T(k) \\
& =T(f)-\widehat{f}_{\tau, T}=0 .
\end{aligned}
$$


By Proposition 2.5, $(E, \tau)$ has Dini's property and so $\lim _{g \in G} \supset g=0$ with respect to $\tau$. Fix, $V \in \mathcal{U}_{\tau}$ and consider $W \in \mathcal{U}_{\tau}$ such that $W+W+W+W$ $\subset V$; then there exists $W_{0} \in \mathcal{U}_{\tau}$ such that for every $W_{1} \in \mathcal{U}_{\tau}$ with $W_{1} \subset W_{0}$,

$$
T(f)-\inf _{\substack{k \in H \\(f-k)^{+} \in W_{1}}} T(k) \in W .
$$

In particular, set $V_{1}:=W_{0} \cap V$;

$$
T(f)-\inf _{\substack{k \in H \\(f-k)^{+} \in V_{1}}} T(k) \in W .
$$

Consider now the subset $\widetilde{G}$ of $E$ consisting of all functions of the form

$$
\inf _{1 \leq i \leq n} T\left(k_{i}\right)-\inf _{\substack{k \in H \\(f-k)^{+} \in V_{1}}} T(k),
$$

with $n \in \mathbb{N}$ and $k_{1}, \ldots, k_{n} \in H,\left(f-k_{i}\right)^{+} \in V_{1}$ for every $i=1, \ldots, n$. Clearly, $\widetilde{G}$ is filtering decreasing and $\inf _{g \in \widetilde{G}} g=0$ because

$$
\begin{aligned}
& \quad \inf _{\substack{k \in H \\
(f-k)^{+} \in V_{1}}} T(k) \\
& =\inf _{n \geq 1} \inf _{1 \leq i \leq n}\left\{T\left(k_{i}\right) \mid k_{1}, \ldots, k_{n} \in H,\left(f-k_{i}\right)^{+} \in V_{1} \text { for every } i=1, \ldots, n\right\} .
\end{aligned}
$$

Thus, $\lim _{g \in \widetilde{G}} \leq g=0$ with respect to $\tau$ and, hence, there exist $n \in \mathbb{N}$ and $k_{1}, \ldots, k_{n} \in H$, with $\left(f-k_{i}\right)^{+} \in V_{1}$ for every $i=1, \ldots, n$, such that

$$
\inf _{1 \leq i \leq n} T\left(k_{i}\right)-\inf _{\substack{k \in H \\(f-k)^{+} \in V_{1}}} T(k) \in W .
$$

Therefore, by taking (1) into account, it follows that

$$
\begin{aligned}
& T(f)-\inf _{1 \leq i \leq n} T\left(k_{i}\right) \\
& =T(f)-\inf _{\substack{k \in H \\
(f-k)^{+} \in V_{1}}} T(k)+\inf _{\substack{k \in H \\
(f-k)^{+} \in V_{1}}} T(k)-\inf _{1 \leq i \leq n} T\left(k_{i}\right) \in W+W .
\end{aligned}
$$

By applying a similar reasoning to $-f$, there exist $V_{2} \in \mathcal{U}_{\tau}, V_{2} \subset V, m \in \mathbb{N}$, and $k_{1}^{\prime}, \ldots, k_{m}^{\prime} \in H$, with $\left(k_{j}^{\prime}-f\right)^{+} \in V_{2}$ for every $j=1, \ldots, m$, such that

$$
\sup _{1 \leq j \leq m} T\left(k_{j}^{\prime}\right)-T(f) \in W+W .
$$

From (2) and (3), it follows that

$$
\inf _{1 \leq i \leq n} T\left(k_{i}\right)-\sup _{1 \leq j \leq m} T\left(k_{j}^{\prime}\right) \in V
$$

and this completes the proof.

(b) $\Rightarrow$ (a). Fix $f \in E$; to show that $f \in \widehat{H}_{\tau, T}$, we shall apply Corollary 3.6. To this end, consider $x \in X$ and $\mu \in M_{\tau, x}^{T}(H)$. Since the mapping 
$g \in E \mapsto \int g d \mu$ is $\tau$-continuous, given $\varepsilon>0$, there exists $V \in \mathcal{U}_{\tau}$ such that for every $g \in V$,

$$
\left|\int g d \mu\right| \leq \varepsilon
$$

Moreover, by assumption, $T \in \mathcal{L}_{\tau, \tau_{\mathrm{s}}}^{+}(E)$ and hence by property (ii) of Definition 2.2 there exists $W \in \mathcal{U}_{\tau}$ such that for every $g \in W$,

$$
|T(g)(x)| \leq \varepsilon
$$

Finally, since the positive linear form $\delta_{x}: E \rightarrow \mathbb{R}$ defined by $\delta_{x}(f):=f(x)$ $(f \in E)$ is $\tau$-continuous, there exists $U \in \mathcal{U}_{\tau}$ such that for every $g \in U$,

$$
|g(x)| \leq \varepsilon .
$$

Set $V_{1}:=V \cap W \cap U$; then there exist $n, m \in \mathbb{N}$ and $k_{1}, \ldots, k_{n}, k_{1}^{\prime}, \ldots, k_{m}^{\prime} \in$ $H$, with $\left(f-k_{i}\right)^{+} \in V_{1}$ for every $i=1, \ldots, n$ and $\left(k_{j}^{\prime}-f\right)^{+} \in V_{1}$ for every $j=1, \ldots, m$, such that

$$
\inf _{1 \leq i \leq n} T\left(k_{i}\right)-\sup _{1 \leq j \leq m} T\left(k_{j}^{\prime}\right) \in V_{1} .
$$

From (1) it follows that for every $i=1, \ldots, n$,

$$
\int f d \mu-\int k_{i} d \mu \leq \int\left(f-k_{i}\right)^{+} d \mu \leq \varepsilon
$$

and, thus,

$$
\int f d \mu \leq \inf _{1 \leq i \leq n} T\left(k_{i}\right)(x)+\varepsilon .
$$

By applying a similar reasoning, we also get

$$
\sup _{1 \leq j \leq m} T\left(k_{j}^{\prime}\right)(x)-\varepsilon \leq \int f d \mu .
$$

Moreover, from (2) it follows that

$$
\sup _{1 \leq j \leq m} T\left(k_{j}^{\prime}\right)(x)-\varepsilon \leq T(f)(x) \leq \inf _{1 \leq i \leq n} T\left(k_{i}\right)(x)+\varepsilon .
$$

Therefore, taking (3) into account, we have

$$
\left|\int f d \mu-T(f)(x)\right| \leq 2 \varepsilon+\inf _{1 \leq i \leq n} T\left(k_{i}\right)(x)-\sup _{1 \leq j \leq m} T\left(k_{j}^{\prime}\right)(x) \leq 3 \varepsilon
$$

and, since $\varepsilon>0$ was arbitrarily chosen, we get $\int f d \mu=T(f)(x)$, and the proof is complete.

Corollary 3.8. Let $(E, \tau)$ be a regular vector lattice on a locally compact Hausdorff space $X, H$ a subspace of $E$ and $T \in \mathcal{L}_{\tau, \tau_{\mathrm{s}}}^{+}(E)$. Then

(i) $\widehat{H}_{\tau, T}$ is closed in $(E, \tau)$.

(ii) If $T \in \mathcal{L}_{\tau}^{+}(E)$, and we denote by $\mathcal{R}(T(H))$ the vector sublattice of $E$ generated by $T(H)$, then

$$
T\left(\widehat{H}_{\tau, T}\right) \subset \overline{\mathcal{R}(T(H))} .
$$


Therefore, if $\widehat{H}_{\tau, T}=E$ and $T$ is onto (or, more generally, if $T\left(\widehat{H}_{\tau, T}\right)$ $=E)$, then $\overline{\mathcal{R}(T(H))}=E$.

Proof. (i) Given $g \in \overline{\widehat{H}}_{\tau, T}$ and $W \in \mathcal{U}_{\tau}$, choose $V \in \mathcal{U}_{\tau}$ such that $V+V \subset W$. There exists $f \in \widehat{H}_{\tau, T}$ such that $f-g \in V$ and hence, by Theorem 3.7, there exist $k_{1}, \ldots, k_{n}, k_{1}^{\prime}, \ldots, k_{m}^{\prime} \in H$ satisfying (i) and (ii) of that theorem with respect to $V$.

For every $i=1, \ldots, n$ and $j=1, \ldots, m$ we get

$$
\begin{aligned}
& \left(g-k_{i}\right)^{+} \leq(g-f)^{+}+\left(f-k_{i}\right)^{+} \in V+V \subset W, \\
& \left(k_{j}^{\prime}-g\right)^{+} \leq\left(k_{j}^{\prime}-f\right)^{+}+(f-g)^{+} \in V+V \subset W .
\end{aligned}
$$

Therefore, $\left(g-k_{i}\right)^{+} \in W,\left(k_{j}^{\prime}-g\right)^{+} \in W$ and $\inf _{1 \leq i \leq n} T\left(k_{i}\right)-\sup _{1 \leq j \leq m} T\left(k_{j}^{\prime}\right)$ $\in W$ and hence $g \in \widehat{H}_{\tau, T}$ by Theorem 3.7.

(ii) Consider $f \in \widehat{H}_{\tau, T}$ and fix $W \in \mathcal{U}_{\tau}$. Choose $U, V \in \mathcal{U}_{\tau}$ such that $U \subset V, T(U) \subset V$ and $V+V+V \subset W$. By Theorem 3.7, we can also consider $k_{1}, \ldots, k_{n}, k_{1}^{\prime}, \ldots, k_{m}^{\prime}$ such that

$$
\begin{aligned}
\left(f-k_{i}\right)^{+} \in U \quad \text { and } & \left(k_{j}^{\prime}-f\right)^{+} \in U \quad \text { for } i=1, \ldots, n \text { and } j=1, \ldots, m, \\
& \inf _{1 \leq i \leq n} T\left(k_{i}\right)-\sup _{1 \leq j \leq m} T\left(k_{j}^{\prime}\right) \in U .
\end{aligned}
$$

For given $i=1, \ldots, n$ and $j=1, \ldots, m$, we get

$$
f-k_{i} \leq \sup _{1 \leq i \leq n}\left(f-k_{i}\right)^{+}=: u \in U, \quad k_{j}^{\prime}-f \leq \sup _{1 \leq j \leq m}\left(k_{j}^{\prime}-f\right)^{+}=: v \in U .
$$

Therefore,

$$
T(f)-\inf _{1 \leq i \leq n} T\left(k_{i}\right) \leq T(u)
$$

and

$$
\begin{aligned}
\inf _{1 \leq i \leq n} T\left(k_{i}\right)-T(f) & =\inf _{1 \leq i \leq n} T\left(k_{i}\right)-\sup _{1 \leq j \leq m} T\left(k_{j}^{\prime}\right)+\sup _{1 \leq j \leq m} T\left(k_{j}^{\prime}\right)-T(f) \\
& \leq \inf _{1 \leq i \leq n} T\left(k_{i}\right)-\sup _{1 \leq j \leq m} T\left(k_{j}^{\prime}\right)+T(v) .
\end{aligned}
$$

Accordingly,

$$
\begin{aligned}
& \left|T(f)-\inf _{1 \leq i \leq n} T\left(k_{i}\right)\right| \\
& \quad \leq T(u)+\left|\inf _{1 \leq i \leq n} T\left(k_{i}\right)-\sup _{1 \leq j \leq m} T\left(k_{j}^{\prime}\right)\right|+T(v) \in V+V+V \subset W .
\end{aligned}
$$

This shows that $T(f) \in \overline{\mathcal{R}(T(H))}$.

4. Generalized affine functions with respect to the identity operator. The results of the last section also hold true when $T$ is the identity operator $I$ on $E$. This particular case is worth a separate consideration. 
For the sake of simplicity, we adopt suitable symbols for this case. The enveloping functions (3.11) and (3.12) will be denoted by $\widehat{f}_{\tau}$ and $\breve{f}_{\tau}$ respectively. Analogously, we use the symbols $\widehat{H}_{\tau}, M_{\tau, x}(H)$ and $\partial_{\tau, H} X$ to denote the subsets (3.14), (3.15) and (3.17), respectively, for $T=I$.

Thus, for every $f \in E$ and $x \in X$ we have

$$
\widehat{f}_{\tau}(x)=\sup _{U \in \mathcal{U}_{\tau}} \inf _{\substack{k \in H \\(f-k)^{+} \in U}} k(x), \quad \breve{f}_{\tau}(x)=\inf _{U \in \mathcal{U}_{\tau}} \sup _{\substack{h \in H \\(h-f)^{+} \in U}} h(x) .
$$

Clearly,

$$
\begin{array}{ll}
\check{f}_{\tau} \leq f \leq \widehat{f}_{\tau} & \text { for every } f \in E, \\
\check{h}_{\tau}=h=\widehat{h}_{\tau} & \text { for every } h \in H .
\end{array}
$$

Moreover,

$$
\widehat{H}_{\tau}=\left\{f \in E \mid \check{f}_{\tau}=f=\widehat{f}_{\tau}\right\},
$$

and its elements will be called generalized $H$-affine functions with respect to the topology $\tau$.

Furthermore, for every $x \in X$,

$$
M_{\tau, x}(H)=\left\{\mu \in M_{\tau, E}^{+}(X) \mid \int h d \mu=h(x) \text { for every } h \in H\right\} .
$$

Every $\mu \in M_{\tau, x}(H)$ is said to be an H-representing measure for $x$ with respect to the topology $\tau$.

Finally, the set

$$
\begin{array}{r}
\partial_{\tau, H} X=\left\{x \in X \mid M_{\tau, x}(H)=\left\{\varepsilon_{x}\right\}\right\} \\
=\left\{x \in X \mid \text { if } \mu \in M_{\tau, E}^{+}(X) \text { and } \int h d \mu=h(x) \text { for every } h \in H,\right. \\
\text { then } \left.\int f d \mu=f(x) \text { for every } f \in E\right\}
\end{array}
$$

is said to be the Choquet boundary of $H$ with respect to the topology $\tau$. Clearly, from Remark 3.3, it follows that $\partial_{\tau, H} X=\partial_{\tau, \widehat{H}_{\tau}} X$.

Moreover, from Theorem 3.4, we get the following result.

TheOREM 4.1. Let $(E, \tau)$ be a regular vector lattice on a locally compact Hausdorff space $X$, and $H$ a subspace of $E$. Then for every $x \in X$ and $f \in E$,

$$
] \breve{f}_{\tau}(x), \widehat{f}_{\tau}(x)\left[\subset\left\{\int f d \mu \mid \mu \in M_{\tau, x}(H)\right\} \subset\left[\check{f}_{\tau}(x), \widehat{f}_{\tau}(x)\right] .\right.
$$

The analogues of Corollary 3.5, Corollary 3.6 and Theorem 3.7 are stated below.

COROLlary 4.2. Under the assumptions of Theorem 4.1,

$$
\partial_{\tau, H} X=\left\{x \in X \mid \check{f}_{\tau}(x)=f(x)=\widehat{f}_{\tau}(x) \text { for every } f \in E\right\} .
$$


In particular, if $\partial_{\tau, H} X \neq \emptyset$, then $\widehat{f}_{\tau}(x)<+\infty$ and $\check{f}_{\tau}(x)>-\infty$ for every $x \in \partial_{\tau, H} X$ and $f \in E$.

THEOREM 4.3. Let $(E, \tau)$ a regular vector lattice on a locally compact Hausdorff space $X, H$ a subspace of $E$, and $f \in E$. Then the following statements are equivalent:

(a) $f \in \widehat{H}_{\tau}$.

(b) For every $V \in \mathcal{U}_{\tau}$ there exist $n, m \in \mathbb{N}$ and $k_{1}, \ldots, k_{n}, k_{1}^{\prime}, \ldots, k_{m}^{\prime} \in H$ such that:

(i) $\left(f-k_{i}\right)^{+} \in V$ for every $i=1, \ldots, n$ and $\left(k_{j}^{\prime}-f\right)^{+} \in V$ for every $j=1, \ldots, m$;

(ii) $\inf _{1 \leq i \leq n} k_{i}-\sup _{1 \leq j \leq m} k_{j}^{\prime} \in V$.

(c) $\int f d \mu=f(x)$ for every $x \in X$ and $\mu \in M_{\tau, x}(H)$.

Moreover, $\partial_{\tau, H} X=X$ if and only if $\widehat{H}_{\tau}=E$.

In the case where $T=I$, Corollary 3.8 can be restated as follows.

COROLlary 4.4. Let $(E, \tau)$ be a regular vector lattice on a locally compact Hausdorff space $X, H$ a subspace of $E$, and $\mathcal{R}(H)$ the vector sublattice of $E$ generated by $H$. Then:

(i) $\widehat{H}_{\tau}$ is closed in $(E, \tau)$ and $\widehat{H}_{\tau} \subset \overline{\mathcal{R}(H)}$.

(ii) If $\widehat{H}_{\tau}=E$, i.e. $\partial_{\tau, H} X=X$, then $\overline{\mathcal{R}(H)}=E$.

A simple consequence of Corollary 4.4 is the following Stone-Weierstrasstype theorem that could be compared with [9, Theorem 2.6], [12, Theorem 5], [18, Theorem 4.4], [19, Theorem 1.19], [27, Corollary 2.2].

We recall that a subset $H$ of $C(X, \mathbb{R})$ separates linearly the points of $X$ if for any distinct $x, y \in X$, there exist $h, k \in H$ such that $h(x) k(y) \neq$ $h(y) k(x)$.

It is easy to show that $H$ separates linearly the points of $X$ if and only if for any distinct $x, y \in X$ and $\alpha \in \mathbb{R}$ there exists $h \in H$ such that $h(x) \neq$ $\alpha h(y)$.

In particular, if $H=H_{+}-H_{+}$(and, hence, in particular, if $H$ is a vector sublattice of $C(X, \mathbb{R}))$ then $H$ separates linearly the points of $X$ if and only if for any $x, y \in X$ and $\alpha \geq 0$ there exists $h \in H$ (which can be chosen to be positive) such that $h(x) \neq \alpha h(y)$.

Finally, if $H$ separates linearly the points of $X$, then for every $x \in X$ there exists $h \in H$ such that $h(x) \neq 0$.

COROLlary 4.5. Let $(E, \tau)$ be a regular vector lattice on a locally compact Hausdorff space $X$, and $H$ a linear sublattice of $E$ linearly separating the points of $X$. Then $H$ is dense in $(E, \tau)$. 
Proof. In this case $\mathcal{R}(H)=H$ and hence, according to Corollary 4.4, it is enough to show that $\partial_{\tau, H} X=X$.

Let $x_{0} \in X$ and $\mu \in M_{\tau, x_{0}}(H)$. Given $h_{0} \in H$ such that $h_{0} \geq 0$ and $h_{0}\left(x_{0}\right)=1$, for every $h \in H$ with $h \geq 0$ we get

$$
\int\left|h-h\left(x_{0}\right) h_{0}\right| d \mu=0
$$

because $\left|h-h\left(x_{0}\right) h_{0}\right| \in H$. Therefore

$$
\operatorname{supp}(\mu) \subset \bigcap_{\substack{h \in H \\ h \geq 0}}\left\{x \in X \mid h(x)=h\left(x_{0}\right) h_{0}(x)\right\}=\left\{x_{0}\right\}
$$

because $H$ linearly separates the points of $X$.

The above inclusion implies that there exists $\lambda \geq 0$ such that $\mu=\lambda \varepsilon_{x_{0}}$. Since

$$
1=h_{0}\left(x_{0}\right)=\int h_{0} d \mu=\lambda h_{0}\left(x_{0}\right)=\lambda,
$$

we have, indeed, $\mu=\varepsilon_{x_{0}}$, and this shows that $x_{0} \in \partial_{\tau, H} X$.

Theorems 4.3 and 3.7 can be fruitfully combined to study the space of generalized affine functions with respect to a lattice homomorphism $T$ : $E \rightarrow E$.

We recall that, by Lemma 2.4, for a given lattice homomorphism $T \in$ $\mathcal{L}_{\tau, \tau_{\mathrm{s}}}^{+}(E)$, there exist $\lambda \in \mathbb{R}^{X}$ with $\lambda \geq 0$ and $\varphi: X \rightarrow X$ such that

$$
T(f)=\lambda(f \circ \varphi) \quad(f \in E) .
$$

Corollary 4.6. Let $X$ be a locally compact Hausdorff space, $(E, \tau)$ a regular vector lattice on $X, H$ a subspace of $E$, and $T \in \mathcal{L}_{\tau}^{+}(E)$ a lattice homomorphism. Then

$$
\widehat{H}_{\tau} \subset \widehat{H}_{\tau, T} .
$$

Proof. Let $f \in \widehat{H}_{\tau}$. To show that $f \in \widehat{H}_{\tau, T}$ we shall verify statement (b) of Theorem 3.7. Given $V \in \mathcal{U}_{\tau}$, since $T$ is $(\tau, \tau)$-continuous, there exists $U \in \mathcal{U}_{\tau}$ with $U \subset V$ such that

$$
T(g) \in V \quad \text { for every } g \in U \text {. }
$$

By Theorem 4.3 , there exist $k_{1}, \ldots, k_{n}, k_{1}^{\prime}, \ldots, k_{m}^{\prime} \in H$ such that

$$
\begin{array}{ll}
\left(f-k_{i}\right)^{+} \in U & \text { for every } i=1, \ldots, n, \\
\left(k_{j}^{\prime}-f\right)^{+} \in U & \text { for every } j=1, \ldots, m
\end{array}
$$

and

$$
\inf _{1 \leq i \leq n} k_{i}-\sup _{1 \leq j \leq m} k_{j}^{\prime} \in U .
$$

Therefore, from (1) it follows that

and this completes the proof.

$$
\inf _{1 \leq i \leq n} T\left(k_{i}\right)-\sup _{1 \leq j \leq m} T\left(k_{j}^{\prime}\right) \in V
$$


Acknowledgements. The authors wish to thank the anonymous referee for the careful reading and several useful suggestions. In particular, the authors are indebted for her/his suggestion to simplify the initial axioms contained in Definition 2.2, which has led the authors to prove Theorem 2.6 and Proposition 2.8 .

\section{References}

[1] C. D. Aliprantis and O. Burkinshaw, Locally Solid Riesz Spaces, Academic Press, New York, 1978.

[2] F. Altomare, Théorèmes de convergence de type Korovkin relativement à une application linéaire positive, Boll. Un. Mat. Ital. B (5) 16 (1979), 1013-1031.

[3] F. Altomare and M. Campiti, Korovkin-Type Approximation Theory and its Applications, de Gruyter Stud. Math. 17, de Gruyter, Berlin, 1994.

[4] F. Altomare and M. Cappelletti Montano, Affine projections on adapted subalgebras of continuous functions, Positivity, to appear.

[5] F. Altomare and S. Diomede, Contractive Korovkin subsets in weighted spaces of continuous functions, Rend. Circ. Mat. Palermo (2) 50 (2001), 547-568.

[6] - - - Positive operators and approximation in function spaces on completely regular spaces, Int. J. Math. Math. Sci. 61 (2003), 3841-3871.

[7] B. Anger and J. Lembcke, Hahn-Banach type theorems for hypolinear functionals, Math. Ann. 209 (1974), 127-151.

[8] H. Bauer, Theorems of Korovkin type for adapted spaces, Ann. Inst. Fourier (Grenoble) 23 (1973), no. 4, 245-260.

[9] - Convergence of monotone operators, Math. Z. 136 (1974), 315-330.

[10] H. Bauer and K. Donner, Korovkin approximation in $C_{0}(X)$, Math. Ann. 236 (1978), 225-237.

[11] C. Berg, J. P. R. Christensen and P. Ressel, Harmonic Analysis on Semigroups, Springer, New York, 1984.

[12] R. C. Buck, Bounded continuous functions on a locally compact space, Michigan Math. J. 5 (1958), 95-104.

[13] G. Choquet, Lectures on Analysis, Vols. I-III, Benjamin, New York, 1969.

[14] K. Donner, Extension of Positive Operators and Korovkin Theorems, Lecture Notes in Math. 904, Springer, Berlin, 1982.

[15] H. O. Flösser, A Korovkin-type theorem in locally convex M-spaces, Proc. Amer. Math. Soc. 72 (1978), 456-460.

[16] -, Korovkin-closures of finite sets, Arch. Math. (Basel) 32 (1979), 600-608.

[17] - Smooth functionals on lattices of continuous functions, in: Functional Analysis, Holomorphy and Approximation Theory (Rio de Janeiro, 1979), Lecture Notes in Pure Appl. Math. 83, Dekker, New York, 1983, 159-190.

[18] H. O. Flösser, R. Irmisch and W. Roth, Infimum-stable convex cones and approximation, Proc. London Math. Soc. 42 (1981), 104-120.

[19] A. Goullet de Rugy, Une classe d'espaces de Banach réticulés, Math. Z. 144 (1975), 217-238.

[20] M. W. Grossman, Korovkin theorems for adapted spaces with respect to a positive operator, Math. Ann. 220 (1976), 253-262.

[21] G. Jameson, Ordered Linear Spaces, Lecture Notes in Math. 141, Springer, Berlin, 1970. 
[22] P. P. Korovkin, On convergence of linear positive operators in the space of continuous functions, Dokl. Akad. Nauk SSSR 90 (1953), 961-964 (in Russian).

[23] G. G. Lorentz, M. von Golitschek and Y. Makovoz, Constructive Approximation, Grundlehren Math. Wiss. 304, Springer, Berlin, 1996.

[24] G. Mokobodzki and D. Sibony, Cônes adaptés de fonctions continues et théorie du potentiel, in: Séminaire Choquet 1966/67, Initiation à l'Analyse, Fasc. 1, Exp. 5, Secrétariat Mathématique, Paris, 1968, 35 pp.

[25] L. Nachbin, Elements of Approximation Theory, Van Nostrand, Princeton, NJ, 1967.

[26] J. B. Prolla, Approximation of Vector-Valued Functions, Math. Stud. 25, NorthHolland, Amsterdam, 1977.

[27] W. Roth, A Korovkin type theorem for weighted spaces of continuous functions, Bull. Austral. Math. Soc. 55 (1997), 239-248.

[28] H. H. Schaefer and M. P. Wolff, Topological Vector Spaces, Springer, Berlin, 1999.

[29] Yu. A. Shashkin, Convergence of positive linear operators in the space of continuous functions, Dokl. Akad. Nauk SSSR 131 (1960), 525-527 (in Russian); English transl.: Soviet Math. Dokl. 1 (1960), 303-305.

[30] - Korovkin systems in spaces of continuous functions, Izv. Akad. Nauk SSSR Ser. Mat. 26 (1962), 495-512 (in Russian); English transl.: Amer. Math. Soc. Transl. Ser. 2, 54 (1966), 125-144.

[31] D. Sibony, Cônes de fonctions et potentiels, lecture notes, McGill Univ., Montreal, 1968.

[32] W. H. Summers, Dual spaces of weighted spaces, Trans. Amer. Math. Soc. 151 (1970), 323-333.

Department of Mathematics

University of Bari

Campus Universitario

Via E. Orabona, 4

70125 Bari, Italy

E-mail: altomare@dm.uniba.it montano@dm.uniba.it

Received September 22, 2004

Revised version July 21, 2005 